\title{
Régénération artificielle des pessières noires à éricacées : effets du scarifiage, de la fertilisation et du type de plants après 10 ans
}

\author{
par Nelson Thiffault ${ }^{1,2}$, Guillaume Cyr $^{1}$, Guy Prégent ${ }^{1}$, Robert Jobidon ${ }^{1}$ et Lise Charette ${ }^{1}$
}

\begin{abstract}
À la suite de perturbations naturelles ou anthropiques, certaines stations de la forêt boréale québécoise sont sujettes à l'envahissement par des éricacées. Cet envahissement provoque souvent un blocage de la succession pouvant perdurer plusieurs décennies. Avec l'objectif de développer des stratégies qui assureront le succès d'établissement des plantations sur ces stations, une étude d'interactions a été entreprise en 1991. Des plants d'épinette noire, de pin gris et de mélèze laricin ont été mis en terre. Des traitements de types de plants, de scarifiage et de fertilisation ont été appliqués selon un dispositif en blocs aléatoires à parcelles partagées. Nous avons effectué un suivi de la croissance en hauteur sur une période de 10 ans et évalué la surface terrière ainsi que la survie à la dixième année. Nous observons une majorité de réponses additives. Les résultats indiquent qu'il est essentiel, du point de vue de la croissance en hauteur et en surface terrière, que les stations de pessières noires à éricacées de l'Est du Québec soient préparées mécaniquement. Le scarifiage a permis une meilleure expression de l'indice de qualité de station qui implique une modification durable des caractéristiques du microsite et qui dépasse un effet à court terme sur la minéralisation et la disponibilité des éléments nutritifs. La fertilisation améliore la croissance des plants, mais de manière moins importante que le scarifiage. Le choix de l'espèce a un impact notable sur la productivité de la station reboisée. Après 10 ans, le choix du type de plants utilisé a une influence marginale sur le succès d'établissement de ces plantations, lorsque comparé aux gains issus des traitements de scarifiage ou de fertilisation.
\end{abstract}

Mots-clés : Kalmia angustifolia, éricacée, survie, croissance, régénération, épinette noire, pin gris, mélèze laricin

Site invasion by ericaceous shrubs after perturbation, either natural or human, is a major concern on some sites of the boreal forest of Québec. A dense cover of ericaceous shrubs often induces a conifer "growth check," which can last several decades. An interaction study was initiated in 1991 with the general objective of developing silvicultural strategies to ensure plantation success on such sites. Black spruce, jack pine, and tamarack seedlings were planted. Stock type, scarification, and fertilization treatments were organised in a randomized block split plot design. We measured seedling total height periodically over a ten-year period and assessed basal area and survival ten years after plantation. Most responses to treatments were additive. Results indicated that mechanical soil scarification is of major importance to ensure seedling growth, both in height and basal area, on spruce-ericaceous shrub sites of northeastern Québec. Scarification allowed a better expression of the site index, implying a sustainable impact on microsite characteristics that is greater than shortterm effects on mineralization and nutrient availability. Fertilization positively influenced seedling growth, but less than soil scarification. Species choice had a strong impact on site productivity. Over a ten-year period, stock types only had a minor influence on plantation success, when compared to scarification or fertilization effects.

Key words: Kalmia angustifolia, ericaceous shrubs, survival, growth, regeneration, black spruce, jack pine, tamarack

\section{Introduction}

Au Québec, le reboisement est pratiqué comme complément à la régénération naturelle dans les cas où cette dernière ne produit pas un nombre adéquat de semis dans un délai acceptable. Or, une certaine proportion des stations reboisées présente de faibles croissances juvéniles, lesquelles compromettent les objectifs de production. Par exemple, si certaines plantes de la famille des éricacées (notamment le Kalmia angustifolia L.) sont présentes sous le couvert forestier, on assiste souvent à une prolifération rapide de ces espèces, à la suite de la coupe forestière (Page 1970, Mallik 1995). L'envahissement des parterres de coupe par les éricacées conduit le plus souvent à un blocage de la succession forestière, lequel peut durer plusieurs décennies (Damman 1971) et ainsi compromettre la croissance d'une régénération forestière (voir Mallik 2003).

L'inhibition de la croissance des plants de conifères en présence de Kalmia est le résultat de plusieurs processus, lesquels agissent seuls ou en interaction. Ces phénomènes comprennent le ralentissement de la minéralisation des litières

\footnotetext{
${ }^{1}$ Direction de la recherche forestière, Ministère des Ressources naturelles, de la Faune et des Parcs, 2700 Einstein, Sainte-Foy, Québec, G1P 3W8.

${ }^{2}$ courriel : nelson.thiffault@mrnfp.gouv.qc.ca.
}

qui entraîne l'accumulation d'humus, la forte compétition pour les éléments nutritifs et potentiellement des effets allélopathiques (Jobidon 1995, Titus et al. 1995).

Comme méthode de maîtrise du Kalmia, plusieurs auteurs (Richardson 1981, Mallik 1994, Titus et al. 1995, Yamasaki et al. 2002, Thiffault 2003) recommandent l'utilisation d'une intense préparation mécanique du terrain qui mélange la matière organique au sol minéral. L'exposition du sol minéral qui en résulte semble créer une barrière à l'expansion du Kalmia (Titus et al. 1995) et ainsi procurer aux plants de conifères des microsites exempts de son influence. Ceci a été vérifié par Prévost $(1996,1997)$ qui a noté une diminution du recouvrement en éricacées à la suite du traitement et par Yamasaki et al. (1998) qui ont établi une relation inversement proportionnelle entre la croissance de l'épinette noire et sa proximité aux plants de Kalmia. En forêt boréale, le scarifiage a également des effets bénéfiques sur les caractéristiques du sol. Il permet d'en améliorer les conditions de température, d'humidité, de fertilité et de densité, qui deviennent alors plus favorables à l'établissement des plants mis en terre (Örlander et al. 1990, Prévost 1992).

La diminution de la disponibilité des éléments nutritifs et la forte compétition pour ceux-ci sont des facteurs importants des 
processus d'interférence associés aux éricacées (Thiffault 2003, Titus et al. 1995). La fertilisation offre l'opportunité d'atténuer ces effets (Taylor et Tabbush 1990). Par exemple, Bradley et al. (2000) démontrent que l'application d'engrais permet d'améliorer la qualité nutritionnelle de stations en Colombie-Britannique envahies par le salal (Gaultheria shallon Pursh.), une autre éricacée.

Les dimensions initiales des plants mis en terre peuvent influencer la croissance à court (Jobidon et al. 1998) et moyen termes (Jobidon et al. 2003). Grâce à leurs masses racinaire et foliaire plus importantes, les gros plants profitent d'un potentiel de photosynthèse plus importante que celle des petits plants (Grossnickle 2000), ce qui accroît leur potentiel à occuper rapidement le microsite de plantation. La compréhension des interactions entre des traitements sylvicoles et des produits de pépinière est essentielle au développement et au raffinement de stratégies sylvicoles qui permettront de réduire, voire d'éliminer, la période de stagnation de croissance des plants mis en terre sur des stations vulnérables à l'envahissement des éricacées.

Nos objectifs sont d'évaluer, pour trois espèces de conifère, l'effet du scarifiage, de la fertilisation, du type de plants et de leurs interactions sur les dimensions et la survie des plants, dix ans après traitement et sur les profils de croissance en hauteur sur dix ans.

\section{Matériels et méthodes Secteur d'étude}

Le secteur d'étude est situé au Québec, dans la région écologique 5g-T (Côte de Baie-Comeau-Sept-Îles) de la sapinière à bouleau blanc de l'Est (Saucier et al. 1998). La température annuelle moyenne est de 0 à $1^{\circ} \mathrm{C}$, les précipitations utiles varient de 300 à $325 \mathrm{~mm} \mathrm{an}^{-1}$ et on compte de 170 à 180 jours sans gel annuellement. La région est caractérisée par un relief accidenté, morcelé et fracturé, dont $75 \%$ de la superficie est occupée par des surfaces rocheuses dénudées ou des tills indifférenciés minces (Robitaille et Saucier 1998). Le secteur supportait des peuplements d'épinette noire âgés de plus de 120 ans, d'une hauteur moyenne variant de $17 \mathrm{~m}$ à $22 \mathrm{~m}$. Les peuplements ont été récoltés par coupe totale entre 1978 et 1984. Le sol est un podzol humo-ferrique (Soil Classification Working Group 1998) et est recouvert d'un humus de type mor d'environ $20 \mathrm{~cm}$ d'épaisseur.

\section{Dispositif expérimental}

Un dispositif à parcelles partagées trois fois en blocs aléatoires a été établi sur des territoires de coupe non régénérés et envahis par un couvert dense et uniforme d'éricacées (Ledum groenlandicum Oeder et Kalmia). Le dispositif compte deux blocs (répétitions), chacun partagé en quatre parcelles principales de $60 \mathrm{~m} \times 50 \mathrm{~m}$, auxquelles a été attribué de façon aléatoire l'un des traitements de scarifiage suivants : (i) témoin (sans scarifiage: T); (ii) scarifiage manuel à l'aide d'une tarière motorisée (SM); (iii) scarifiage mécanisé avec un scarificateur à cônes de type Wadell (SW); et (iv) scarifiage mécanisé avec une herse forestière Le Crabe (SH). Le scarifiage a eu lieu en 1990. Les parcelles principales ont été divisées en sous-parcelles de $60 \mathrm{~m} \times 10 \mathrm{~m}$. Chacune a été reboisée, en 1991, avec 150 plants de l'une des espèces suivantes : épinette noire (Picea mariana (Mill.) BSP), pin gris (Pinus banksiana Lamb.) et mélèze laricin (Larix laricina (Du Roi) K. Koch). L'attribution des espèces à chaque sous-parcelle s'est faite de manière aléatoire. Des plants de pin sylvestre (Pinus sylvestris L.) et de mélèze de Sibérie (Larix siberica Ledeb.) ont également été plantés mais les résultats pour ces espèces ne sont pas présentés. Pour chaque sousparcelle, la moitié des plants (sous-sous-parcelle) a reçu une fertilisation au moment de leur mise en terre, alors que l'autre moitié n'a pas été fertilisée. L'attribution du traitement de fertilisation à chaque sous-sous-parcelle a été faite de manière aléatoire. L'engrais utilisé était de forme granulaire et a été appliqué au pied de chacun des plants à un taux de $100 \mathrm{~g} \mathrm{plant}^{-}$ ${ }^{1}\left(8 \% \mathrm{~N}\right.$ total, $24 \% \mathrm{P}_{2} \mathrm{O}_{5}, 12 \% \mathrm{~K}_{2} \mathrm{O}$, ce qui équivaut à 8,0 , 10,4 et $10,0 \mathrm{~g}$ de N, P, K élémentaires, respectivement). Pour chaque combinaison de scarifiage $\times$ espèce $\times$ fertilisation ainsi formée, trois types de plants $(1+0)$ ont été utilisés et distribués de façon aléatoire au niveau de la sous-sous-sous-parcelle : (i) 25 plants produits en récipients multipots de 67 cavités de $50 \mathrm{~cm}^{3}$ chacune (RC67-50); (ii) 25 plants produits en récipients multipots de 45 cavités de $110 \mathrm{~cm}^{3}$ chacune (RC45-110); et (iii) 25 plants Jiffy (JIFFY). Les plants ont été produits selon les normes en vigueur au Québec (Tétreault et al. 1990) à partir de semences provenant de la région d'étude. Le traitement de scarifiage SM est manquant pour l'un des blocs. Les plants ont été mis en terre en respectant un espacement de $2 \mathrm{~m} \times 2 \mathrm{~m}\left(2500\right.$ plants $\left.^{-1}\right)$.

La hauteur de tous les plants a été mesurée aux années 2, 3, 6 et 10 après la plantation. Nous avons mesuré le diamètre à hauteur de poitrine (dhp, mesuré à 1,30 m au-dessus du sol) des plants à la dixième saison de croissance. Pour chacun des blocs, nous avons calculé la surface terrière à 10 ans $\left(G_{t}\right.$, en $\mathrm{cm}^{2} / 0.01 \mathrm{ha}$ ) de l'unité expérimentale (combinaison de scarifiage $\times$ espèce $\times$ fertilisation $\times$ type de plants) où le dhp prend la valeur 0 si le plant n'avait pas atteint $1,30 \mathrm{~m}$ au moment de la prise de mesure. Lors du mesurage, nous avons noté la survie des plants. La variable Survie = 1 si le plant est vivant au moment de la prise de mesure et Survie $=0$ si ce n'est pas le cas.

\section{Analyses statistiques}

Hauteur, surface terrière et survie à 10 ans

Nous avons effectué les analyses séparément pour chacune des trois essences. Le plan analysé correspondait à un plan en blocs aléatoires à parcelles doublement partagées. Nous avons évalué les effets des traitements sur la hauteur et la surface terrière par analyses de la variance (ANOVA), à l'aide de la procédure MIXED du logiciel SAS, version 8.02 (SAS Institute Inc., Cary, NC). Nous avons utilisé les moyennes ajustées pour le non équilibrage ainsi que pour prendre en compte la variance des différents éléments aléatoires du modèle. Au besoin, nous avons effectué une transformation sur la variable dépendante afin de respecter les hypothèses de normalité et d'homogénéité de la variance des erreurs. Pour ces cas, les moyennes ajustées présentées sont celles issues de transformations inverses, une fois qu'une correction ait été effectuée pour minimiser le biais causé par les transformations (Ung et Végiard 1988). Afin de modéliser le caractère binomial de la survie, nous avons retenu une approche de modélisation linéaire généralisée, que nous avons effectuée à l'aide de la macro procédure GLIMMIX de SAS, en retenant la fonction de lien logit (Littell et al. 1996).

\section{Profil de croissance}

Nous avons examiné le profil temporel (années 2, 3, 6 et 10 après la plantation) de la croissance en hauteur selon une 
analyse de la variance à mesures répétées (ANOVAR), laquelle fait appel à l'option REPEATED de la procédure MIXED de SAS. Nous avons décomposé l'effet Année par contrastes en ses composantes linéaire, quadratique et manque d'ajustement. Nous présentons et interprétons les résultats issus de ces contrastes seulement lorsque l'effet Année et les interactions qui lui sont associées sont déclarés significatifs. Nous avons basé le choix d'un modèle de covariance entre les années de mesure sur le critère d'Akaike (Milliken et Johnson 2001). Dans le cas du mélèze laricin, nous avons choisi une structure de covariance autorégressive d'ordre 1 pour des données inégalement espacées (spatial power law) (voir Littell et al. 1996). Pour les autres essences, nous avons retenu des structures de covariance sans structure particulière.

Pour l'ensemble des analyses, nous avons utilisé un seuil de $\alpha=0,05$ pour déclarer un effet significatif. De plus, en ce qui concerne le scarifiage, nous avons construit des contrastes orthogonaux a priori afin de répondre aux questions suivantes : $(i)$ y a-t-il un avantage à scarifier, peu importe la méthode? (ii) lorsqu'il y a scarifiage, y a-t-il une différence de l'effectuer de façon mécanique ou manuelle? et (iii) lorsqu'il y a scarifiage mécanisé, y a-t-il prépondérance d'une méthode par rapport à l'autre?

Pour les contrastes qui testent l'avantage d'un traitement ou d'une combinaison de traitements comparativement à un autre, nous avons spécifié une hypothèse alternative unilatérale. Nous avons procédé de la même manière pour la fertilisation. Nous avons inclus le traitement SM dans les analyses malgré qu'il soit manquant pour l'un des blocs, en utilisant les fonctionnalités de la procédure MIXED de SAS en présence de données manquantes. Il en découle une moins grande précision pour tester le contraste (ii) ainsi qu'une inférence nécessairement limitée au cadre de l'expérience. Nous avons calculé les degrés de liberté associés selon l'approximation de Satterthwaite.

\section{Résultats \\ Hauteur après 10 ans et profil de croissance Épinette noire}

Le scarifiage et la fertilisation influencent la hauteur après 10 ans des plants d'épinette noire, mais leurs effets respectifs ne sont pas du même ordre de grandeur selon le type de plants mis en terre (Tableau 1). Globalement, il y a un avantage à scarifier $(72,7 \mathrm{~cm}$ contre $43,7 \mathrm{~cm})$, peu importe la méthode. Nous notons des interactions significatives pour les contrastes qui comparent, par type de plants, les méthodes mécanisées à la méthode manuelle et le scarifiage Wadell au scarifiage Le Crabe. Pour un type de plants donné, par exemple le RC45-110, il n'y a pas suffisamment d'évidence statistique pour conclure à une différence entre les approches mécanisées $(94,2 \mathrm{~cm})$ et l'approche manuelle $(56,6 \mathrm{~cm})$ de même qu'entre les scarificateurs Wadell $(85,6 \mathrm{~cm})$ et Le Crabe $(103,7 \mathrm{~cm})$. Sur un terrain scarifié de façon mécanique, les plants RC45-110 atteignent, après 10 ans, une hauteur moyenne supérieure à celles des plants RC67-50 et JIFFY ( $94,2 \mathrm{~cm}$ contre $81,3 \mathrm{~cm}$ et $70,7 \mathrm{~cm}$, respectivement). Les plants RC67-50 et JIFFY atteignent respectivement des hauteurs moyennes de $22 \%$ et $42 \%$ supérieures lorsque fertilisés, alors qu'aucun effet significatif de la fertilisation n'est noté pour le RC45-110. En l'absence de fertilisation, les plants RC45-110 et JIFFY $(64,0 \mathrm{~cm}$ et $58,1 \mathrm{~cm}$, respectivement) atteignent une hauteur moyenne supérieure à celle des RC67-50 (49,4 cm). Cette différence s'estompe en présence de fertilisation.
Pour cette espèce, les profils de croissance en hauteur de l'interaction scarifiage $\times$ fertilisation ne sont pas parallèles (Tableau 2). L'examen de l'interaction révèle le non-parallélisme des profils scarifié et témoin lesquels interagissent avec les niveaux de fertilisation : l'effet de la fertilisation s'estompe dans le temps en absence de scarifiage (Figure 1). Les profils des scarifiages faits de façons mécanisée et manuelle ne sont pas parallèles : le léger avantage initial en hauteur moyenne des plants des parielles SM s'estompe et s'inverse entre les années 3 et 6 au profit des plants mis en terre dans les parcelles scarifiées de façon mécanique. Le non-parallélisme est également observé entre les profils des scarificateurs Wadell et Le Crabe. Les profils des types de plants sont, pour leur part, parallèles. L'interprétation des contrastes linéaire, quadratique, et manque d'ajustement n'est pas directe pour cette essence, compte tenu que l'analyse est effectuée sur des données transformées par logarithme. C'est pourquoi nous nous limitons à l'interprétation générale.

\section{Pin gris}

Après 10 ans, la hauteur des plants de pin gris n'est pas influencée par le type de plants mis en terre (Tableau 1). Toutes méthodes confondues, le scarifiage donne une hauteur moyenne des plants supérieure à celle des plants des parcelles témoins $(157,4 \mathrm{~cm}$ contre $87,2 \mathrm{~cm})$. Les plants mis en terre dans les parcelles scarifiées de façon mécanique sont plus hauts de $66 \%$ en moyenne, après 10 ans, que les plants mis en terre dans les parcelles scarifiées de façon manuelle. La fertilisation confère un avantage à tous les types de plants, peu importe le type de scarifiage pratiqué $(155,1 \mathrm{~cm}$ contre $121,7 \mathrm{~cm})$.

Les profils de croissance en hauteur afférents aux différentes méthodes de scarifiage ne sont pas parallèles. L'amplitude entre les profils du scarifiage, toutes méthodes confondues, et du témoin augmente généralement de façon constante avec les années mais des déviations à cette tendance sont observées (Tableau 2 et Figure 1). Il en est de même pour les profils des scarifiages exécutés par voies mécanisée et manuelle; ceux-ci sont similaires pendant les premières années, puis se séparent par la suite avec un avantage pour l'approche mécanisée. Les profils de croissance des niveaux de fertilisation ne sont pas parallèles; celui des parcelles fertilisées s'éloigne de celui des parcelles non fertilisées à un rythme généralement constant mais des déviations à cette tendance sont également notées. Les types de plants présentent toutefois des profils parallèles, qui coïncident tout au long de la période d'étude.

\section{Mélèze laricin}

Pour le mélèze laricin, le scarifiage permet de doubler la hauteur moyenne après 10 ans (gain de $117 \%$ ), et toutes les méthodes de scarifiage sont équivalentes (Tableau 1). Les plants fertilisés atteignent une hauteur moyenne supérieure à celle des plants non fertilisés ( $238,6 \mathrm{~cm}$ contre $174,5 \mathrm{~cm}$ ). Les plants RC45-110 et JIFFY atteignent respectivement $231,4 \mathrm{~cm}$ et $215,9 \mathrm{~cm}$ et sont plus grands que les plants RC67-50 $(172,3 \mathrm{~cm})$ après 10 ans.

Les profils de croissance en hauteur selon les différents traitements de scarifiage, de fertilisation et de types de plants ne sont pas parallèles (Tableau 2 et Figure 1). Le profil du scarifiage, toutes méthodes confondues, est supérieur à celui du témoin, et l'amplitude entre les deux suit une progression qui s'accentue avec les années. L'amplitude entre les profils des scarifiages faits de façons mécanisée et manuelle augmente de façon 
Tableau 1. Résultat de 10 ans de l'analyse de la variance (valeur de $p$ ) sur la hauteur des trois espèces à l'étude (EPN = épinette noire; PIG = pin gris; MEL = mélèze laricin)

\begin{tabular}{|c|c|c|c|c|c|}
\hline Source de variation (fixe) ${ }^{a}$ & d.l.n. & d.l.d. ${ }^{c}$ & $E N^{d}$ & PIG $^{\mathbf{e}}$ & MEL $^{f}$ \\
\hline Scarifiage & 3 & 3,0 & 0,077 & $<0,001$ & 0,051 \\
\hline SM, SW, SH contre T & 1 & 3,0 & 0,018 & $<0,001$ & 0,013 \\
\hline SW, SH contre SM & 1 & 3,0 & 0,156 & $<0,001$ & 0,091 \\
\hline SW contre $\mathrm{SH}$ & 1 & 3,0 & 0,894 & 0,093 & 0,752 \\
\hline Fertilisation & 1 & 3,0 & 0,024 & 0,001 & 0,022 \\
\hline Scarifiage $\times$ Fertilisation & 3 & 3,0 & 0,851 & 0,975 & 0,454 \\
\hline Type & 2 & 11,3 & 0,003 & 0,499 & $<0,001$ \\
\hline Scarifiage $\times$ Type & 6 & 11,4 & 0,015 & 0,317 & 0,543 \\
\hline$(\mathrm{SM}, \mathrm{SW}, \mathrm{SH}$ contre T) $\times$ Type & 2 & 12,1 & 0,102 & & \\
\hline$(\mathrm{SW}, \mathrm{SH}$ contre $\mathrm{SM}) \times$ Type & 2 & 10,6 & 0,046 & & \\
\hline$(\mathrm{SW}$ contre $\mathrm{SH}) \times$ Type & 2 & 11,5 & 0,018 & & \\
\hline Fertilisation $\times$ Type & 2 & 11,3 & 0,045 & 0,232 & 0,859 \\
\hline Scarifiage $\times$ Fertilisation $\times$ Type & 6 & 11,4 & 0,207 & 0,911 & 0,391 \\
\hline
\end{tabular}

aSM = Scarifiage manuel SW = Scarifiage Wadell $; \mathrm{SH}=$ Scarifiage herse Le Crabe $; \mathrm{T}=$ Témoin, sans scarifiage Type = Type de plants.

bd.l.n. = degrés de liberté du numérateur.

$\mathbf{c}_{\text {d.l.d. }}=$ degrés de liberté du dénominateur selon l'approximation de Satterthwaite.

dAnalyse effectuée sur $\ln$ (hauteur).

eAnalyse effectuée sur $\sqrt{\text { hauteur }}$; d.1.d. = 17,9 pour tout effet étant donné des composantes de variance estimées nulles.

$\mathbf{f}_{\text {d.l.d. }}=12,1$ pour l'effet Type et les interactions avec Type.

constante dans le temps, alors que les profils des scarificateurs Wadell et Le Crabe sont parallèles et confondus tout au long de l'étude. L'examen des contrastes pour les niveaux de fertilisation démontre que l'amplitude entre les profils s'accentue de façon constante dans le temps, avec des moyennes plus élevées en présence de fertilisation. Il en est de même pour les profils de croissance en hauteur des différents types de plants, pour lesquels les profils des RC45-110 et JIFFY présentent les moyennes les plus élevées.

\section{Surface terrière et survie \\ Épinette noire}

Tous traitements confondus, moins de $10 \%$ des plants vivants après 10 ans atteignent $1,30 \mathrm{~m}$ et possèdent donc un dhp mesurable. Les valeurs de surface terrière sont nulles pour toutes les tiges des parcelles témoins et scarifiées de façon manuelle. Cela implique une variabilité nulle pour ces traitements; ils sont exclus de l'analyse de la variance. Aucun effet significatif des autres traitements de scarifiage et du traitement de fertilisation n'est noté (données non-illustrées). Pour ces cas, les trois types de plants présentent, après 10 ans, des surfaces terrières moyennes similaires.

L'interaction triple observée pour la survie après 10 ans (Tableau 3) est analysée pour chaque combinaison de traitements à l'aide d'un tableau des taux de survie moyens (données non-illustrées). Compte tenu que la présence de taux de survie moyens de $100 \%$ dans certaines combinaisons de facteurs rend le test exact de l'interaction triple impossible par l'approche de modélisation retenue, nous avons effectué un test approximatif en imputant la valeur de «mort » pour un plant de chaque unité expérimentale dont le taux de survie est de $100 \%$. Cette modification des données ne permet cependant pas de tester avec rigueur la décomposition de l'interaction. L'interprétation sommaire de cette interaction indique qu'en absence de scarifiage, la fertilisation diminuerait le taux de survie ( $72 \%$ contre $87 \%$ ). Les RC67-50 affichent les plus bas taux de survie six fois sur huit (pour chacune des huit combinaisons de scarifiage $\times$ fertilisation) $(79 \%$ en moyenne contre $88 \%$ pour chacun des deux autres types de plants).

\section{Pin gris}

Seuls le scarifiage et la fertilisation ont un effet significatif sur la surface terrière du pin gris après 10 ans et ces effets sont additifs (Tableau 3). Le scarifiage, toutes méthodes confondues, permet de multiplier par six la surface terrière moyenne des parcelles, par rapport à celle des parcelles témoins $\left(38,9 \mathrm{~cm}^{2} / 0,01\right.$ ha contre $6,1 \mathrm{~cm}^{2} / 0,01 \mathrm{ha}$ ). Le scarifiage par voie mécanique a un effet du même ordre de grandeur par rapport au scarifiage manuel $\left(60,8 \mathrm{~cm}^{2} / 0,01\right.$ ha contre $\left.10,7 \mathrm{~cm}^{2} / 0,01 \mathrm{ha}\right)$. Les plants fertilisés cumulent une surface terrière moyenne 1,7 fois supérieure à celle des plants non fertilisés.

La fertilisation réduit le pourcentage de survie des plants, le faisant passer de $91 \%$ à $79 \%$, mais seulement dans les parcelles scarifiées. Les plants RC67-50 (79\%) présentent un pourcentage de survie inférieur à ceux des RC45-110 (87\%) et des JIFFY (90\%), ces deux derniers étant équivalents.

\section{Mélèze laricin}

Pour le mélèze laricin, les analyses démontrent que le scarifiage procure un avantage, en regard de la surface terrière, mais que cet avantage n'est pas le même selon le type de plants mis en terre (Tableau 3). Les plants RC45-110 réagissent mieux (de $8,0 \mathrm{~cm}^{2} / 0,01$ ha à $115,3 \mathrm{~cm}^{2} / 0,01 \mathrm{ha}$ ), en moyenne, que les plantes JIFFY (de $9,3 \mathrm{~cm}^{2} / 0,01$ ha à $90,8 \mathrm{~cm}^{2} / 0,01 \mathrm{ha}$ ), et les plants RC67-50 (de $0,8 \mathrm{~cm}^{2} / 0,01$ ha à $\left.51,0 \mathrm{~cm}^{2} / 0,01\right)$. Indépendamment du type de plants, le scarifiage par voie mécanisée produit une surface terrière moyenne supérieure à celle issue du scarifiage par voie manuelle $\left(118,5 \mathrm{~cm}^{2} / 0,01\right.$ ha contre $20,0 \mathrm{~cm}^{2} / 0,01 \mathrm{ha}$ ). Dans tous les cas, la fertilisation permet de doubler la surface terrière moyenne après 10 ans.

Le scarifiage a un effet sur la survie des plants et celui-ci dépend du type de plants mis en terre (Tableau 3). Pour les plants RC45110 et RC67-50, le pourcentage de survie est plus élevé sur un sol scarifié, toutes méthodes confondues, que sur sol non scarifié. Sur un sol scarifié par voie mécanique, le taux de survie est plus élevé pour les plants JIFFY ( $89 \%$ ) que pour les plants RC67-50 (81\%), alors que sur un sol scarifié de façon manuelle, le taux de survie est plus élevé pour les plants JIFFY (97\%) que pour les plants RC45-110 (80\%). Les taux 
Tableau 2. Analyse des profils de la hauteur (ANOVAR; valeur de $p$ ) des trois espèces à l'étude, au cours des 10 premières années (EPN = épinette noire; PIG = pin gris; $M E L=$ mélèze laricin)

\begin{tabular}{|c|c|c|c|c|c|}
\hline Source de variation $(\text { fixe })^{\mathrm{a}}$ & d.l.n. ${ }^{b}$ & d.l.d. ${ }^{c}$ & EPN $^{d}$ & PIG $^{e}$ & MEL $^{f}$ \\
\hline Scarifiage & 3 & 3,0 & 0,138 & $<0,001$ & $<0,001$ \\
\hline SM, SW, SH contre T & 1 & 3,0 & 0,023 & $<0,001$ & $<0,001$ \\
\hline SW, SH contre SM & 1 & 3,0 & 0,570 & $<0,001$ & $<0,001$ \\
\hline SW contre SH & 1 & 3,0 & 0,777 & 0,153 & 0,230 \\
\hline Fertilisation & 1 & 2,4 & 0,007 & $<0,001$ & $<0,001$ \\
\hline Scarifiage $\times$ Fertilisation & 3 & 2,4 & 0,812 & 0,495 & 0,027 \\
\hline Type & 2 & 13,0 & 0,002 & 0,414 & $<0,001$ \\
\hline Scarifiage $\times$ Type & 6 & 13,0 & 0,027 & 0,250 & 0,858 \\
\hline Fertilisation $\times$ Type & 2 & 13,0 & 0,130 & 0,181 & 0,813 \\
\hline Scarifiage $\times$ Fertilisation $\times$ Type & 6 & 13,0 & 0,082 & 0,921 & 0,565 \\
\hline Année & 3 & 18,0 & $<0,001$ & $<0,001$ & $<0,001$ \\
\hline Scarifiage $\times$ Année & 9 & 18,0 & $<0,001$ & $<0,001$ & $<0,001$ \\
\hline$(\mathrm{SM}, \mathrm{SW}, \mathrm{SH}$ contre T) $\times$ Année & 3 & 18,0 & $<0,001$ & $<0,001$ & $<0,001$ \\
\hline Linéaire & 1 & 18,0 & $<0,001$ & $<0,001$ & $<0,001$ \\
\hline Quadratique & 1 & 18,0 & $<0,001$ & 0,088 & 0,003 \\
\hline Manque d'ajustement & 1 & 18,0 & 0,214 & 0,045 & 0,940 \\
\hline$(\mathrm{SW}, \mathrm{SH}$ contre $\mathrm{SM}) \times$ Année & 3 & 18,0 & $<0,001$ & $<0,001$ & $<0,001$ \\
\hline Linéaire & 1 & 18,0 & $<0,001$ & $<0,001$ & $<0,001$ \\
\hline Quadratique & 1 & 18,0 & 0,155 & 0,066 & 0,325 \\
\hline Manque d'ajustement & 1 & 18,0 & $<0,001$ & $<0,001$ & 0,870 \\
\hline$(\mathrm{SW}$ contre $\mathrm{SH}) \times$ Année & 3 & 18,0 & 0,002 & 0,334 & 0,915 \\
\hline Linéaire & 1 & 18,0 & 0,132 & & \\
\hline Quadratique & 1 & 18,0 & 0,013 & & \\
\hline Manque d'ajustement & 1 & 18,0 & 0,085 & & \\
\hline Fertilisation $\times$ Année & 3 & 18,0 & $<0,001$ & $<0,001$ & $<0,001$ \\
\hline Linéaire & 1 & 18,0 & 0,043 & 0,042 & $<0,001$ \\
\hline Quadratique & 1 & 18,0 & 0,072 & 0,071 & 0,696 \\
\hline Manque d'ajustement & 1 & 18,0 & 0,828 & 0,002 & 0,777 \\
\hline Scarifiage $\times$ Fertilisation $\times$ Année & 9 & 18,0 & 0,038 & 0,319 & 0,181 \\
\hline$(\mathrm{SM}, \mathrm{SW}, \mathrm{SH}$ contre $\mathrm{T}) \times$ Fertilisation $\times$ Année & 3 & 18,0 & 0,004 & & \\
\hline Linéaire & 1 & 18,0 & 0,773 & & \\
\hline Quadratique & 1 & 18,0 & 0,003 & & \\
\hline Manque d'ajustement & 1 & 18,0 & 0,287 & & \\
\hline$(\mathrm{SW}, \mathrm{SH}$ contre $\mathrm{SM}) \times$ Fertilisation $\times$ Année & 3 & 18,0 & 0,831 & & \\
\hline$(\mathrm{SW}$ contre $\mathrm{SH}) \times$ Fertilisation $\times$ Année & 3 & 18,0 & 0,289 & & \\
\hline Type $\times$ Année & 6 & 18,0 & 0,076 & 0,101 & 0,002 \\
\hline Linéaire & 2 & 18,0 & & & $<0,001$ \\
\hline Quadratique & 2 & 18,0 & & & 0,512 \\
\hline Manque d'ajustement & 2 & 18,0 & & & 0,992 \\
\hline Scarifiage $\times$ Type $\times$ Année & 18 & 18,0 & 0,625 & 0,504 & 0,993 \\
\hline Fertilisation $\times$ Type $\times$ Année & 6 & 18,0 & 0,942 & 0,400 & 0,996 \\
\hline Scarifiage $\times$ Fertilisation $\times$ Type $\times$ Année & 18 & 18,0 & 0,551 & 0,793 & 0,996 \\
\hline
\end{tabular}

avoir le Tableau 1 pour la signification des abréviations.

bd.l.n. = degrés de liberté du numérateur.

$\mathbf{c}_{\text {d.l.d. }}=$ degrés de liberté du dénominateur selon l'approximation de Satterthwaite.

dAnalyse effectuée sur le ln(hauteur).

$\mathbf{e}_{\text {d.l.d. }}=18,0$ pour tout effet étant donné les composantes de variance estimées nulles.

f.l.d. varient entre 12,4 et 44,2 selon les effets.

de survie des niveaux de fertilisation ne présentent pas de différence significative.

\section{Discussion}

Globalement, le scarifiage du sol a des effets bénéfiques sur la croissance des plants. Le gain en hauteur lié à l'utilisation du scarifiage s'accentue dans le temps, sinon à une puissance 2 ou supérieure pour toutes les essences, au moins de façon constante. Cette influence positive du travail du sol sur la croissance était déjà présente cinq ans après l'application des traitements sur ces stations (Veilleux et Lévesque 1997), et elle est généralement observée en milieu boréal (Örlander et al. 1990, Prévost 1992, Sutton 1993, Macadam et Bedford 1998). Le scarifiage a permis une meilleure expression de l'indice de qualité de station (IQS), qui exprime la relation entre la hauteur des arbres et leur âge. Les effets bénéfiques du scarifiage sur la croissance des plants peuvent, en général, être attribués à l'impact du traitement sur la température, la teneur en eau du sol, le régime nutritif du sol et la végétation de compétition (Örlander et al. 1990, Prévost 1992), dans ce cas-ci principalement le Kalmia (Prévost 1996, 1997; Yamasaki et al. 1998). L'impact positif du scarifiage que nous avons observé, qui s'est maintenu et dans certains cas accru dans le temps, implique une modification durable des caractéristiques du microsite qui dépasse un effet à court terme sur la minéralisation et la disponibilité des éléments nutritifs (Morris et Lowery 1988).

Les effets plus modestes du scarifiage manuel par rapport au scarifiage mécanique sont le résultat d'une perturbation moins forte et moins durable de l'humus. De plus, le scarifiage manuel crée un microsite de plantation circulaire dont le rayon (environ $20 \mathrm{~cm}$ ) limite l'éloignement significatif des arbustes de Kalmia et de Ledum. Les deux méthodes de scarifiage par voie mécanique donnent généralement des résultats similaires. Par ailleurs, ces deux méthodes, et particulièrement le 


\section{Scarifiage}

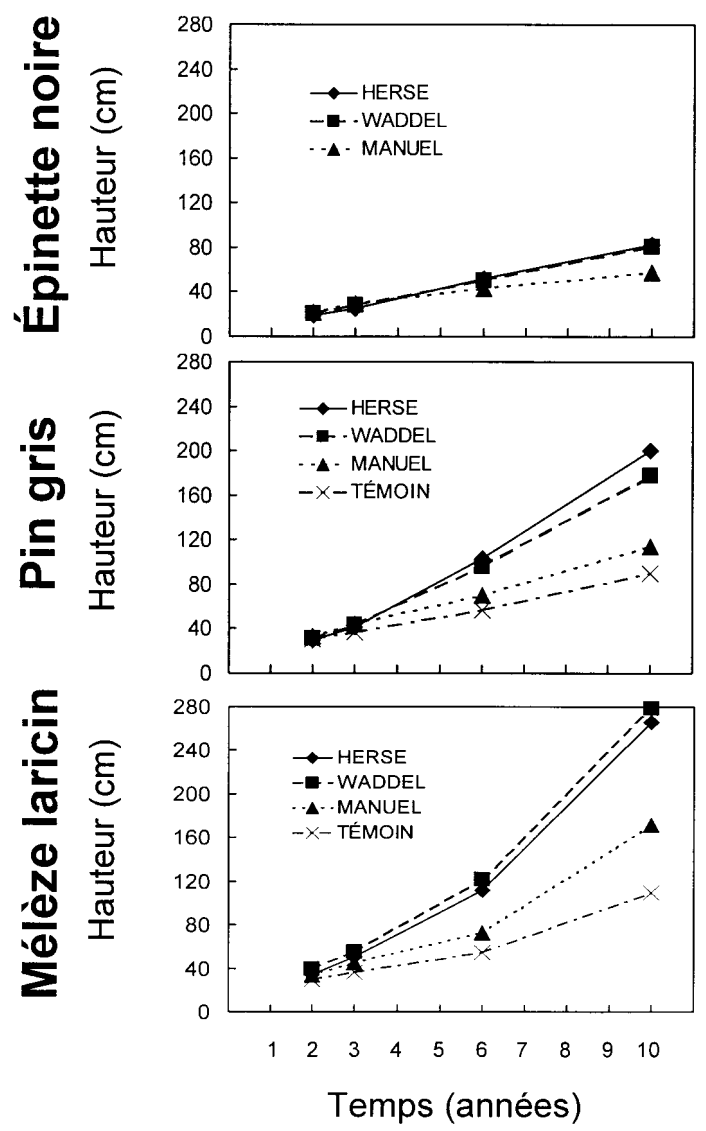

Fertilisation
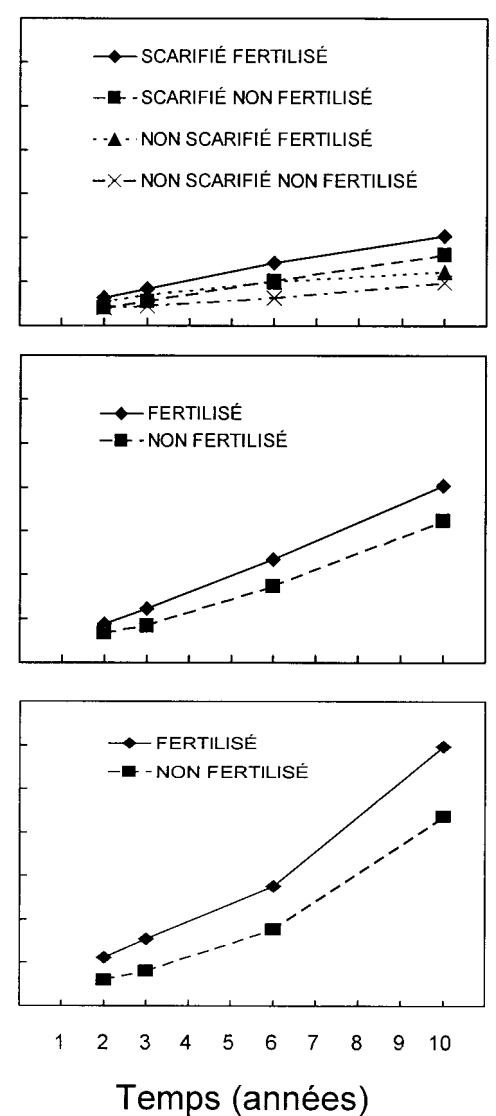

Type de plant

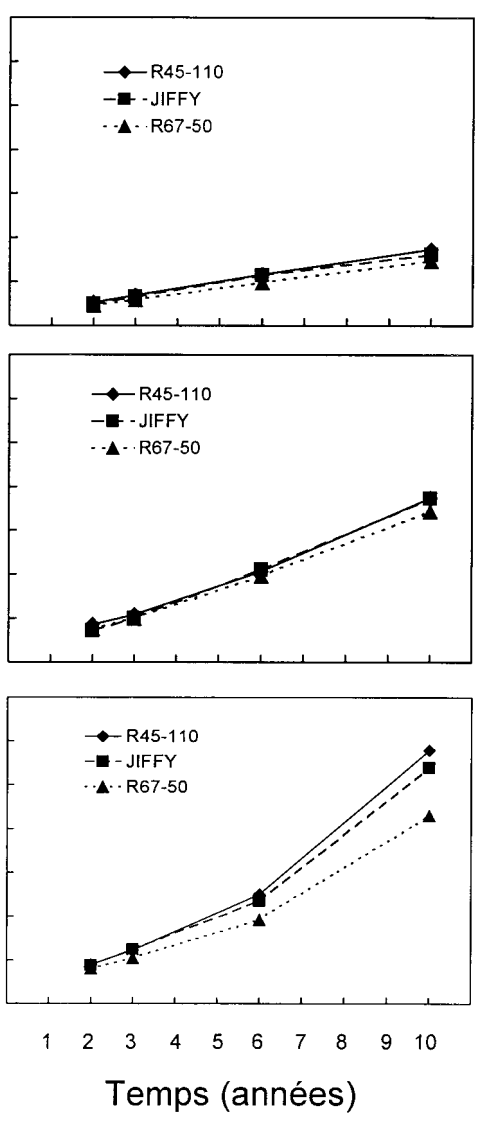

Fig. 1. Effets principaux du scarifiage, de la fertilisation et du type de plant (colonnes) sur le profil de la hauteur pour trois espèces de conifère (rangées) au cours des 10 premières années. Dans le cas de l'épinette noire, l'effet de la fertilisation est présenté selon qu'il y ait eu scarifiage ou non tout en prenant compte l'interaction entre ces deux facteurs.

scarifiage de type Wadell, sont bien adaptées à la préparation mécanique des sols minces (Ryans et Sutherland 2001) car les appareils utilisés peuvent être ajustés en fonction de la profondeur du sol.

Concernant les effets de la fertilisation, et à l'instar de l'influence du scarifiage, les tendances observées après cinq ans (Veilleux et Lévesque 1997) se confirment. Globalement, les plants fertilisés atteignent des dimensions supérieures à celles des plants non fertilisés. L'amplitude entre les profils s'accentue dans le temps. Ces résultats, similaires à ceux obtenus par Blevins et Prescott (2002) sur des stations dominées par le salal, appuient l'hypothèse que l'induction d'un stress nutritif par les éricacées à l'égard des conifères est une cause importante de l'interférence observée (Yamasaki et al. 2002, Thiffault 2003). Sur un site mésique apparemment productif de la forêt boréale québécoise, Paquin et al. (1998) ont noté que la croissance de marcottes et de plants d'épinette noire fertilisés est supérieure à celle de témoins non fertilisés, et ce, sur une station exempte d'éricacées. Ainsi, dans la présente étude, la réaction positive de la croissance des plants à l'ajout d'engrais peut être, d'autre part, le reflet d'une limitation des ressources liée aux caractéristiques du site, sans égard à la végétation arbustive. Le pro- tocole expérimental ne permet pas de distinguer les limitations nutritionnelles causées par la présence d'éricacées de celles inhérentes à la station.

Pour l'ensemble des essences à l'étude, les gains associés à la fertilisation ne peuvent compenser l'absence de scarifiage mécanique. En ce qui a trait à la hauteur après 10 ans, l'avantage du scarifiage mécanique par rapport à la fertilisation est de $14 \%$ en moyenne pour le mélèze laricin et de $20 \%$ en moyenne pour les autres espèces. En absence de scarifiage, la fertilisation au moment de la mise en terre améliore probablement le statut nutritif des plants, lequel se traduit par des croissances accrues par rapport aux témoins non fertilisés. Cependant, la proximité des plantes éricacées ainsi que l'isolation thermique du sol par l'épaisse couche d'humus limitent l'apport des éléments nutritifs et empêchent les plants d'exprimer davantage leur plein potentiel de croissance. Dans le cas de l'épinette noire, l'effet positif de la fertilisation sur la croissance en hauteur s'estompe dans le temps en absence de scarifiage. Dans certains cas, l'ajout d'engrais au moment de la mise en terre a eu un effet négatif sur la survie des plants. Cet effet était présent cinq ans après la plantation (Veilleux et Lévesque 1997). Cela indique que la mortalité est apparue pendant les premières 
Tableau 3. Résultat de 10 ans de l'analyse de la variance (valeur de $p)$ sur la survie $(S)$ et la surface terrière $\left(G_{t}\right)$ des trois espèces à l'étude $(E P N=$ épinette noire; PIG = pin gris; $M E L=$ mélèze laricin)

\begin{tabular}{|c|c|c|c|c|c|c|c|c|c|}
\hline \multirow[t]{2}{*}{ Source de variation (fixe) ${ }^{a}$} & \multicolumn{2}{|c|}{ d.l.n. ${ }^{b}$} & \multicolumn{2}{|c|}{ d.l.d. ${ }^{c}$} & \multirow{2}{*}{$\frac{\text { EPN }^{\mathbf{f}}}{\mathrm{S}}$} & \multicolumn{2}{|c|}{ PIG } & \multicolumn{2}{|c|}{ MEL } \\
\hline & $\mathbf{S}$ & $\mathbf{G}_{\mathbf{t}}$ & $\mathbf{S}^{\mathbf{d}}$ & $G_{t} \mathbf{e}^{\mathbf{e}}$ & & $\mathbf{S}$ & $\mathbf{G}_{\mathrm{t}}{ }^{\mathrm{g}}$ & $\mathbf{S}$ & $G_{t}$ \\
\hline Scarifiage & 3 & 3 & 5,3 & 3 & 0,908 & 0,618 & $<0,001$ & 0,211 & 0,041 \\
\hline SM, SW, SH contre T & 1 & 1 & 5,1 & 3 & 0,250 & 0,298 & $<0,001$ & 0,025 & 0,017 \\
\hline SW, SH contre SM & 1 & 1 & 5,3 & 3 & 0,896 & 0,708 & $<0,001$ & 0,589 & 0,039 \\
\hline SW contre SH & 1 & 1 & 5,5 & 3 & 0,955 & 0,278 & 0,111 & 0,775 & 0,513 \\
\hline Fertilisation & 2 & 2 & 5,3 & 3 & 0,563 & $<0,001$ & 0,035 & 0,131 & 0,012 \\
\hline Scarifiage $\times$ Fertilisation & 3 & 3 & 5,3 & 3 & 0,613 & 0,099 & 0,662 & 0,722 & 0,105 \\
\hline$(\mathrm{SM}, \mathrm{SW}, \mathrm{SH}$ contre $\mathrm{T}) \times$ Fertilisation & 1 & 1 & 5,1 & 3 & & 0,037 & & & \\
\hline$(\mathrm{SW}, \mathrm{SH}$ contre $\mathrm{SM}) \times$ Fertilisation & 1 & 1 & 5,3 & 3 & & 0,403 & & & \\
\hline$(\mathrm{SW}$ contre $\mathrm{SH}) \times$ Fertilisation & 1 & 1 & 5,5 & 3 & & 0,215 & & & \\
\hline Type & 2 & 2 & 1009 & 12 & $<0,001$ & $<0,001$ & 0,245 & $<0,001$ & $<0,001$ \\
\hline Scarifiage $\times$ Type & 6 & 6 & 1010 & 12 & 0,719 & 0,408 & 0,422 & $<0,001$ & 0,018 \\
\hline$(\mathrm{SM}, \mathrm{SW}, \mathrm{SH}$ contre $\mathrm{T}) \times$ Type & 2 & 2 & 1010 & 12 & & & & 0,043 & 0,032 \\
\hline$(\mathrm{SW}, \mathrm{SH}$ contre $\mathrm{SM}) \times$ Type & 2 & 2 & 1010 & 12 & & & & 0,002 & 0,073 \\
\hline$(\mathrm{SW}$ contre $\mathrm{SH}) \times$ Type & 2 & 2 & 1010 & 12 & & & & 0,802 & 0,108 \\
\hline Fertilisation $\times$ Type & 2 & 2 & 1009 & 12 & 0,411 & 0,410 & 0,517 & 0,151 & 0,262 \\
\hline Scarifiage $\times$ Fertilisation $\times$ Type & 6 & 6 & 1010 & 12 & 0,012 & 0,269 & 0,945 & 0,796 & 0,212 \\
\hline
\end{tabular}

avoir le Tableau 1 pour la signification des abréviations.

bd.1.n. = degrés de liberté du numérateur.

$\mathbf{c}_{\text {d.l.d. }}=$ degrés de liberté du dénominateur selon l'approximation de Satterthwaite.

dLes d.l.d. diffèrent quelque peu pour PIG et MEL.

ePour PIG, d.1.d. = 18 pour tout effet étant donné les composantes de variance estimées nulles.

${ }^{\mathbf{f}}$ Aucune analyse effectuée sur $\mathrm{G}_{\mathrm{t}}$, car $\mathrm{G}_{\mathrm{t}}=0$ pour $90 \%$ des plants.

gAnalyse effectuée sur $\sqrt{G}_{\text {t }}$.

années d'établissement des plants. La mortalité des plants à la suite d'une fertilisation au moment de la plantation est également rapportée dans d'autres études. Sept ans après la mise en terre, les plants fertilisés d'épinettes (Picea glauca (Moench) Voss, P. engelmannii (Parry) et leurs hybrides naturels) ont un taux de survie de $18 \%$ inférieur à celui des plants non fertilisés (Simpson et Vyse 1995). À Terre-Neuve sur un site envahi par le Kalmia, la fertilisation au moment de la mise en terre nuit à la survie de l'épinette noire, du pin gris et du mélèze laricin (B.D. Titus ${ }^{3}$, comm. pers.). La combinaison de la présence de sels (l'engrais) et des conditions osmotiques défavorables du sol froid caractéristiques de la forêt boréale, où l'eau est peu disponible et la croissance des racines réduite (Örlander et al. 1990), est la cause probable du phénomène observé (Van Den Driessche 1991).

Le type de plants a une influence constante, mais somme toute limitée, sur la croissance et la survie des différentes espèces. De manière générale, le plant produit en récipients de $45 \mathrm{ca}-$ vités de $110 \mathrm{~cm}^{3}$ atteint les dimensions les plus fortes après 10 ans et conserve à tout le moins dans le temps ses différences de dimensions par rapport aux autres types de plants, comme l'indique le parallélisme des courbes de croissance de deux des trois espèces. Walsh et al. (2002) rapportent également, après trois ans, des profils de croissance parallèles pour des RC45110 et des RC67-50 mis en terre en forêt boréale. Les plants RC45-110, tout en bénéficiant d'un système racinaire plus important, ont une carotte de tourbe d'un volume deux fois supérieur à celui des RC67-50. Le RC45-110 profite de l'importation sur le site d'une quantité totale d'éléments nutritifs plus importante que le RC67-50, ce qui a probablement un effet bénéfique sur la croissance aérienne (Burdett 1990).

\footnotetext{
${ }^{3}$ Chercheur scientifique, Service canadien des forêts, Pacific Forestry Centre, Victoria, C.-B.
}

Les essences étudiées ne présentent pas toutes la même croissance, comme le révèlent les dimensions atteintes après 10 ans (Tableau 4). L'épinette noire est celle dont le rendement est le plus faible. En absence de fertilisation et de scarifiage, l'utilisation des courbes de croissance de Prégent et al. (1996), qui illustrent les rendements des plantations du Québec, permet de prévoir un IQS moyen pour l'épinette noire de 2,43 m (âge de référence de 25 ans) avec le récipient RC45-110. Le scarifiage Le Crabe permet de révéler un IQS de 6,21 m-ce qui permet d'escompter un rendement beaucoup plus intéressant - mais qui se retrouve malgré tout parmi les classes de fertilité les plus faibles des tables de rendement (Prégent et al. 1996). En présence de scarifiage mécanique, traitement qui donne les meilleurs résultats peu importe l'espèce, le mélèze laricin, le pin gris et l'épinette noire atteignent respectivement des hauteurs moyennes de 272,184 et $82 \mathrm{~cm}$, ce qui correspond à des facteurs de l'ordre de 3 et 2 entre les deux premières espèces et l'épinette noire. L'épinette noire s'accommode d'une grande variété de stations (Cauboue et Malenfant 1988), ce qui explique en partie le fait qu'elle soit l'espèce la plus utilisée pour le reboisement au Québec (Parent 2003). Cependant, même sur les stations à mauvais drainage, le mélèze laricin est reconnu pour présenter une croissance qui dépasse largement celles des épinettes et des pins (Hall 1977, 1983). Nos résultats militent en faveur d'une diversification des essences utilisées dans les programmes de reboisement. Indirectement, ils illustrent aussi qu'une station donnée doit être reboisée d'une seule essence ou d'espèces qui affichent un rythme de croissance semblable pour éviter la suppression.

La mesure des dimensions atteintes après 10 ans reflète l'effet global des traitements sur cette période. Or, l'effet des traitements sylvicoles sur la croissance des plants est dynamique; elle évolue notamment selon la croissance de la végétation compétitive (Munson et al. 1993). L'analyse des profils permet de comprendre comment les différences observées la 
Tableau 4. Hauteur moyenne ${ }^{\mathrm{a}}$ des plants RC45-110 des trois espèces selon différentes combinaisons de traitements 10 ans après la mise en terre

\begin{tabular}{lccc}
\hline & & \multicolumn{2}{c}{ Hauteur $(\mathbf{c m})$} \\
\cline { 2 - 4 } Espèce & $\begin{array}{c}\text { Témoin } \\
\text { non fertilisé }\end{array}$ & $\begin{array}{c}\text { Herse } \\
\text { Le Crabe }\end{array}$ & $\begin{array}{c}\text { Témoin } \\
\text { fertilisé }\end{array}$ \\
\hline Épinette noire & 44,2 & 98,7 & \multicolumn{1}{c}{ Fertilisation } \\
Pin gris & 96,8 & 158,5 & 120,4 \\
Mélèze laricin & 119,6 & 231,1 & 112,7 \\
\hline
\end{tabular}

aMoyennes non ajustées, à titre indicatif des effets principaux (à l'exception du type de plant). Les résultats spécifiques issus des ANOVAs sont présentés dans le texte.

dixième année sont apparues dans le temps et ainsi mieux interpréter l'effet des traitements à l'étude. Comme plusieurs des courbes de croissance obtenues dans cette étude présentent un caractère non linéaire, l'analyse de l'effet des traitements après deux ou trois saisons de croissance n'aurait pas permis de prévoir adéquatement l'amplitude de ceux-ci à moyen ou plus long termes. Ces résultats nous incitent à la prudence lors de l'interprétation des effets de traitements sylvicoles sur la productivité d'une plantation au cours des premières années d'établissement. Il faut retenir que les tendances observées en bas âges pourront se maintenir, s'accroître ou s'inverser dans les années à venir.

Cette étude comporte un faible nombre de répétitions (2). De plus, un traitement (SM) est absent de l'un des blocs. Ces facteurs nous obligent à faire preuve de circonspection dans l'inférence des résultats obtenus. Bien que les effets soient clairs, ils demeurent seulement applicables dans les limites des conditions de station à l'étude.

\section{Considérations sylvicoles}

Nos résultats illustrent la nécessité de préparer par voie mécanique les stations de pessières noires à éricacées de l'Est du Québec au préalable à la mise en terre des plants, sans quoi le succès d'établissement de la plantation est grandement compromis. Quoique les taux de survie observés après 10 ans soient relativement élevés, les stations non scarifiées et reboisées ont une croissance faible et ne contribuent que marginalement à la productivité forestière (Tableau 4). Dans le cadre de cette étude, il est clair que la croissance des plants et non leur survie doit guider le choix d'un traitement ou d'une combinaison de traitements. Le choix du type de plants utilisé a une influence mineure sur la croissance et la survie de ces plantations, lorsqu'on le compare aux gains obtenus des traitements de scarifiage. Globalement, la fertilisation confère un avantage aux plants en leur fournissant une source supplémentaire d'éléments nutritifs. Néanmoins, les gains afférents à l'utilisation d'engrais demeurent marginaux par rapport à ceux associés au scarifiage (Tableau 4) et ne sauraient les remplacer. Finalement, l'utilisation des espèces qui offrent les meilleurs potentiels de croissance parmi celles adaptées à ces stations, et compatibles avec les objectifs de production, devrait être renforcée (Thiffault et al. 2003).

\section{Remerciements}

Nous adressons nos sincères remerciements aux personnes qui ont participé à toutes les phases de réalisation de cette étude, depuis son installation jusqu'au dernier mesurage. Il s'agit notamment des gestionnaires de la région administrative de la Côte-Nord et du personnel de l'unité de gestion Hauterive du ministère des Ressources naturelles, de la Faune et des Parcs (MRNFP), en particulier messieurs Jacques Duval, André Ruelland et Yvon Rioux. Nous remercions également messieurs Hervé Lortie, Yvon Lévesque et Jean-Yves Montambault de la Direction de la recherche forestière (DRF) du MRNFP pour la qualité de leur travail technique. Les commentaires de madame Isabelle Auger, messieurs Vincent Roy, Pierre Bélanger et Louis Blais, de la DRF, monsieur Stephen Wyatt, de l'Université Laval, et de deux réviseurs anonymes ont permis d'améliorer ce manuscrit; nous leur en sommes reconnaissants. Nous remercions également monsieur Patrick Moreault pour l'édition de la figure. Monsieur Jean-Marc Veilleux, chercheur retraité du MRNFP, est à l'origine de cette recherche et du dispositif expérimental qui a servi à cette étude.

\section{Bibliographie}

Blevins, L.L. et C. Prescott. 2002. Salal Cedar Hemlock Integrated Research Program : Research Update No 2. University of British Columbia, $62 \mathrm{p}$.

Bradley, R.L., B.D. Titus, C.P. Preston et J. Bennett. 2000. Improvement of nutritional site quality 13 years after single application of fertiliser $\mathrm{N}$ and $\mathrm{P}$ on regenerating cedar-hemlock cutovers on northern Vancouver Island, B.C. Plant Soil 223: 195-206.

Burdett, A.N. 1990. Physiological processes in plantation establishment and the development of specifications for forest planting stock. Can. J. For. Res. 20: 415-427.

Cauboue, M. et D. Malenfant. 1988. Le reboisement au Québec : exigences écologiques des épinettes (Picea), des pins (Pinus) et des mélèzes (Larix) plantés au Québec. Ministère de l'Énergie et des Ressources. 90 p.

Damman, A.W.H. 1971. Effect of vegetation changes on the fertility of a Newfoundland forest site. Ecol. Monogr. 41: 253-270.

Grossnickle, S.C. 2000. Ecophysiology of Northern Spruce Species: The Performance of Planted Seedlings. NRC Research Press, Ottawa, ON. 409 p.

Hall, J.P. 1977. Comparison of the early growth of Larix and Picea in plantations in Newfoundland. Newfoundland Forest Research Center, Fisheries and Environment Canada, Bi-monthly Research Notes Vol. 33, no 2.

Hall, J.P. 1983. Comparison of the growth of larch and other conifers on reforested and afforested sites in Newfoundland. For. Chron. 59: 14-16.

Jobidon, R. 1995. Autécologie de quelques espèces de compétition d'importance pour la régénération forestière au Québec : revue de littérature. Direction de la recherche forestière, Ministère des Ressources naturelles, Mémoire de recherche forestière 117, $180 \mathrm{p}$.

Jobidon, R., L. Charette et P.Y. Bernier. 1998. Initial size and competing vegetation effects on water stress and growth of Picea mariana (Mill.) BSP seedlings planted in three different environments. For. Ecol. Manage. 103: 293-305.

Jobidon, R., V. Roy et G. Cyr. 2003. Net effect of competing vegetation on selected environmental conditions and performance of four spruce seedling stock sizes after eight years in Québec (Canada). Ann. For. Sci. (Sous presse). 
Littell, R.C., G.A. Milliken, W.W. Stroup et R.D. Wolfinger. 1996. SAS System for Mixed Models. SAS Institute Inc., Cary, CA, 633 p. Macadam, A. et L. Bedford. 1998. Mounding in the sub-boreal spruce zone of west-central British Columbia: 8-years results. For. Chron 74: 421-427.

Mallik, A.U. 1994. Autecological response of Kalmia angustifolia to forest types and disturbance regimes. For. Ecol. Manage. 65: 231-249.

Mallik, A.U. 1995. Conversion of temperate forests into heaths: role of ecosystem disturbance and ericaceous plants. Environ. Manage. 19: 675-684.

Mallik, A.U. 2003. Conifer regeneration problems in boreal and temperate forests with ericaceous understory: Role of disturbance, seedbed limitation, and keystone species change. Crit. Rev. Plant Sci. 22: 341-366.

Milliken, G.A. et D.E. Johnson. 2001. Analysis of Messy Data. Volume 3: Analysis of Covariance. Chapman \& Hall, New York. 605 p. Morris, L.A. et R.F. Lowery. 1988. Influence of site preparation on soil conditions affecting stand establishment and tree growth. South J. Appl. For. 12: 170-178.

Munson, A.D., H.A. Margolis et D.G. Brand. 1993. Intensive silvicultural treatment: impact on soil fertility and planted conifer response. Soil Sci. Soc. Am. J. 57: 246-255.

Örlander, G., P. Gemmel et J. Hunt. 1990. Site Preparation: A Swedish Overview. British Columbia Ministry of Forests, FRDA Report 105. $61 \mathrm{p}$.

Page, G. 1970. The development of Kalmia angustifolia on a black spruce cutover in Central Newfoundland. Forest Research Laboratory, Internal Report N-27. 7 p.

Paquin, R., H.A. Margolis et R. Doucet. 1998. Nutrient status and growth of black spruce layers and planted seedlings in response to nutrient addition in the boreal forest of Quebec. Can. J. For. Res. 28: 729-736 Parent, B. 2003. Ressources et industries forestières : portrait statistique édition 2002. Gouvernement du Québec, Ministère des Ressources naturelles, Québec, QC

Prégent, G., V. Bertrand et L. Charette. 1996. Tables préliminaires de rendement pour les plantations d'épinette noire au Québec. Direction de la recherche forestière, Ministère des Ressources naturelles, Mémoire de recherche forestière $118.70 \mathrm{p}$

Prévost, M. 1992. Effets du scarifiage sur les propriétés du sol, la croissance des semis et la compétition : revue des connaissances actuelles et perspectives de recherches au Québec. Ann. Sci. For. 49: 277-296. Prévost, M. 1996. Effets du scarifiage sur les propriétés du sol et l'ensemencement naturel dans une pessière noire à mousses de la forêt boréale québécoise. Can. J. For. Res. 26: 72-86.

Prévost, M. 1997. Effects of scarification on seedbed coverage and natural regeneration after a group seed-tree cutting in a black spruce (Picea mariana) stand. For. Ecol. Manage. 94: 219-231.

Richardson, J. 1981. Black spruce research by the Canadian Forestry Service in Newfoundland. Canadian Forest Service, Newfoundland Forest Research Center, Information Report N-X-206. 36 p.

Robitaille, A. et J.P. Saucier. 1998. Paysages régionaux du Québec méridional. Gouvernement du Québec, Ministère des Ressources naturelles, Québec, QC. 213 p.
Ryans, M. et B.J. Sutherland. 2001. Site Preparation - Mechanical. In R.G. Wagner et S.J. Columbo (éditeurs). Regenerating the Canadian Forest: Principles and Practices for Ontario. pp. 178-199. Fitzhenry \& Whiteside, Markham, ON.

Saucier, J.P., J.F. Bergeron, P. Grondin et A. Robitaille. 1998. Les régions écologiques du Québec méridional (3e version). Ministère des Ressources naturelles du Québec, Supplément de l'Aubelle 124. 12 p. Simpson, D.G. et A. Vyse. 1995. Planting stock performance: site and RGP effects. For. Chron. 71: 739-742.

Soil Classification Working Group. 1998. The Canadian System of Soil Classification. 3rd ed. Agriculture and Agri-Food Canada, Publication $1646.187 \mathrm{p}$.

Sutton, R.F. 1993. Mounding site preparation: a review of european and North American experience. New For. 7: 151-192.

Taylor, C.M.A. et P.M. Tabbush. 1990. Nitrogen deficiency in Sitka spruce plantations. For. Comm. (UK) Bull. 89. 30 p.

Tétreault, J.-P., J.-G. Brouillette et J. Lortie. 1990. Guide d'évaluation de la qualité des plants de reboisement produits en récipients (4e version). Division des pépinières, Service de la production des plants, Ministère des Forêts. 45 p.

Thiffault, N. 2003. L'établissement de plantations de conifères en relation avec le scarifiage et la végétation de compétition. Université Laval, Thèse de doctorat (Ph.D.). $155 \mathrm{p}$.

Thiffault, N., V. Roy, G. Prégent, G. Cyr, R. Jobidon et J. Ménétrier. 2003. La sylviculture des plantations résineuses au Québec. Nat. Can. 127: 63-80.

Titus, B.D., S.S. Sidhu et A.U. Mallik. 1995. A summary of some studies on Kalmia angustifolia L. : a problem species in Newfoundland forestry. Canadian Forest Service, Natural Resources Canada, Information Report N-X-296. 68 p.

Ung, C.-H. et S. Végiard. 1988. Problèmes d'inférence statistique reliés à la transformation logarithmique en régression. Can. J. For. Res 18: 733-738.

Van Den Driessche, R. 1991. Effects of nutrients on stock performance in the forest. In R. Van Den Driessche (éditeur). Mineral nutrition of conifer seedlings. pp. 229-260, CRC Press, Boston, MA.

Veilleux, J.M. et Y. Lévesque. 1997. Régénération artificielle de la pessière noire sur sols minces : Résultats quinquennaux préliminaires des plantations de 1991. Direction de la recherche forestière, Ministère des Ressources naturelles, Rapport interne 426. 34 p.

Walsh, D., J. Allaire et D. Lord. 2002. Performance en plantation de plants d'épinette noire de petites dimensions : rapport d'étape pour la période 2001-2002. Programme de mise en valeur des ressources du milieu forestier (volet 1), Ministère des Ressources naturelles. Consortium de recherche sur la Forêt Boréale Commerciale, Université du Québec à Chicoutimi. $30 \mathrm{p}$.

Yamasaki, S.H., J.W. Fyles, K.N. Egger et B.D. Titus. 1998. The effect of Kalmia angustifolia on the growth, nutrition, and ectomycorrhizal symbiont community of black spruce. For. Ecol. Manage. 105: 197-207.

Yamasaki, S.H., J.W. Fyles et B.D. Titus. 2002. Interactions among Kalmia angustifolia, soil characteristics, and the growth and nutrition of black spruce seedlings in two boreal Newfoundland plantations of contrasting fertility. Can. J. For. Res. 32: 2215-2224. 